\title{
América Latina: los tiempos de la integración real y no solo verbal
}

- Exposición realizada el 17 de junio de 2008 por el Canciller Alejandro Foxley con motivo de la presentación del libro "Los Caminos para la Integración de América Latina» en la Universidad Miguel de Cervantes.

Deseo agradecer la invitación de la Iniversidad Miguel de Cervantes para presentar el libro los Caminos para la Integración de América Latima, editado por el lnstituto Latinoamericano de Relaciones Internacionales. Deseo agradecer, especialmente, al Presidente de su Consejo, don Ciutenbere Martínez y a su Rector, querido amigo y ex colega en el Coblerno de don latricio Aylwin, don Irancisco Cumplido.

Cuando miro los rostros en esta sala, veo un reencuentro con personas con las cuales, en distintos momentos en el tiempo, hemos compartido ma cierta idea, me atrevería a decir -en forma poco diplomatica- una cierta "chifladura" por algunos temas que nos parecen extraordinariamente morivadores, y uno de ellos es cómo construimos un futuro de Chile en conjunto con los paises de la región.

Acá hay personas que han estado en este esfucroo por décadas, ya sea descle el gobicrno, desde institutos de estudios internacionales, desde organismos internacionales, como la CFPAL. Es decir, toda una comunidad de personas que ha puesto mucho esfuerzo, mucha energía, para abrir un camino en un proceso de trabajo, en conjunto con otros paises de la región, para construir un futuro para América latina integrada.

I a publicacion de este libro-que puedo decir que es muy bueno pues me lo lci, de principio a fin, aprovechando un vuelo 
muy largo desde Canadá y California- es muy oportuna, por las razones que menciona Patricio l civa.

No hace un mes que (hile pasó a coupar la l'residencia Pro Témpore del primer año de vida de la Unión de Naciones Sudamericana, que se firmó recién, con Brasilia, hace tres semanas. Se le pidió a la Presidenta Bachelet que encabece este proceso el primer año. Por lo tanto, todas las reflexiones al estilo de las que hay en este libro nos van a ser extremadamente litiles para tratar de impregnarle a esta UNASUR un espíritu que nos permita reconocer los avances y, también, superar las deficiencias de procesos de integracion anteriores.

A ver si esta vez logramos un avance mís significativo respecto de lo que decia recién Patricio Leiva, yue es csta idea de una integración de toda la región, no solo América del Sur. También incluye a América del Norte, América Central, en lo que se refiere a México, y el Caribe.

El título del libro ya es bastante sugerente y podemos concluir -como dice Roberto Pizarro en alguno de los artículosgue la integración es, en realidad, un proceso irrentunciable para la region.

Auncue, siendo realistas, se da con avances, retrocesos, y en un contexto que a veces -a mí por lo menos, que no habia estado en este cargo antes ni en este tema directamente-sorprende con un mumero de conflictos subyacentes en lia region, que hacen que los avances no sean tan significativos como uno quisiera.

P'ero, en todo caso, como dice Osvaldo Sunkel en su trabajo, sabemos que la historia, la cultura, la geografía y otros factores apuntan en una dirección favorable para la integración.

La convicion que me he formado en estos años que he estado en el servicio público y, en particular, ahora, en la Cancilleria, es que ha llegado un momento en el que tenemos que avanzar decididamente en la integracion, corrigiendo con mucha francpuezal y muy directamente los enfocpues que han producido insuficientes resultados.

Porque, querámoslo o no, estamos viviendo tiempos de glohalización. Nos guste o no nos guste, ahi estamos. La globalización no es una proposicion de la cual uno pueda decir "paremos el mundo que me quiero bajur". Esto está. 
Todo el mundo está embarcado en cllo, con mayor o menor entusiasmo.

Esos son los ticmpos que vivimos. Por lo tanto, para los países de América Latina hay una palabra clave que es la "asociatividad" para afrontar juntos este desafío de la globalización. Lo entendieron hace rato los paises del este de Furopa. Lo entendieron los países del este del Asia, cule estín avanzande a través de ASFAN, en sus discusiones con (hina, con fapón, para hacerlo allá en el Asia.

I cos tiempos se nos están agotando en América Latina para dar un salto significativo hacia una integración real. No una integración verbal, de buenlas intenciones discursivas, sino una integración con acciones concretas. Ese creo que es el punto principal porque se da la paradoja que todos conocomos de sobra: una región extremadamente rica con recursos naturales -ahora estamos viviendo un "boom", estamos ell un punto superior del cicto-, y creemos que el futuro es 1 1 futuro sin limites. Pero hay dos pequenos detalles que nos faltan: uno, que los ciclos son ciclos, asi como tiene puntos altos, después tiene puntos bajos; y, segundo, que estames arrastrando una pesada mochila histórica yue es el tema de la pobreza y las desigualdades en América Latina.

Ninguno de nosotros puede sentirse orgulloso de estar viviendo en páses en los cuales la distancia entre los ricos y los pobres es tan abismante. como ocurre en buenas partes de la región.

De repente tenemos la tentación-siempre la hemos tenido en América Latinla, porque tenemos unil ventaja comparativa, dirial como economista- de decir muy buenas piezas oratorias. También tenemos una cicra herencia, quizás descle tiempos de la Colonia espanola, para escribir impecables compendios de leyes.

Otras regiones del mundo crecen más rápido que nosotros en sus procesos de integración. Hacen más cooperación entre sí, atumentan más el comercio, le dan mis importancia a la cohesión social y, il veces, hacen menos discursos y no escriben tantes tratados jurídicos.

Fl proceso de integración en América Latina se inicio casi exactamente en el mismo momento en que se inició en Europa, hace 50 anos. Obviamente, ha habido avances en cl dialogo 
político y en comercio. Pero, comparemos con la integración en ese tiltimo aspecto: cl comercio. Los paises de la Union Furopea, hoy dia, realizan entre el 72 y el 74 por ciento de su comercio total entre cllos, comercio intra-zonal. Los paises de América l atina, después de los mismos 50 años, y después de numerosas inciativas de integración, realizamos, como comercio entre los países de la región, no más de un 17 por ciento del comercio total. 72-74 por ciento contra 17. Creo yue es una cifra bastante llamativa y que ahorra mayores comentarios.

Asia, que comenzó bastante después su proceso de integración, ya está llegando a una cifra del 50 por ciento del comercio intra-zonal.

Nadie puede dejar de reconocer, como dice Manuel Agosin en su trabajo $y$ en otros trabajos anteriores, que el avance de Asia es colosal, es más rápido que el nuestro. Que China, Japón, Corea del Sur, Vietnam y otros países no se detienen y que están convergiendo crecientemente en un esquema de integracion común.

Para no hablar de los otros paises ribereños en el Pacífico como Australia o Nueva Zelandia. Parises que tuvieron una visión de anticipación notable hace 2.5 anos, cuando se cayo cl Commonwealth y dijeron: "¿̇ Ahora qué hacemos si somos unas is las en el medio de la nada? ¿Dóncle estamos en el mundo?". Miraron un mapa y dijeron: "Somos el sur del Asia. Somos austral-Asia" y desde entonces, están concentrando sus fuerzas en integrarse con las economías más dinámicas del mundo, en el Asia.

Situćmonos un poco más en la experiencia europea. Recién estuvimos en la Cumbre de I.ma, Unión Furopea- América Latina y el Caribe, y conversamos este tema con los Jefes de lstado, con los Cancilleres europeos. Porque si miramos las instituciones de la integración de América Latina, no son tan diferentes de las europeas, pero los procesos para construir esas instituciones han sido muy distintos. Para los europeos lo básico, incialmente, era la integración en dos planos: la energía y' la infraestructura. O sea, dos pilares en un proceso de este tipo, como señala Mario Vilalva en su capitulo, y que explica muy bien el por qué, partiendo desde alli, ha sido posible ir avanzando en etapas posteriores que son bastante 
reconocibles, graduales, sin un maximalismo inicial, sino a través de etapas llevadas a cabo, paso a paso, en 50 años.

Recién ahora está Furopa discutiendo, al final de ese proceso, lo que podríamos llamar un Tratado Constitutivo, o un Acuerdo Constitucional que también tiene, 50 años después, avances y retrocesos. El referendo de Irlanda así lo demuestra. Es bastante impresionante que el país más exitoso en los últimos 15 ó 20 años, precisamente por su asociación con la Unión Europea, tenga el ingreso per cápita más alto de Europa.

Pero, nosotros no queremos un Tratado Constitucional, porque, en realidad, tenemos un enfoque distinto. Ha sido un enfoque más formal o, llamémosle, "dc jure", que enfatiza la primacía de los instrumentos legales para alcanzar la integración entre los países. Hemos sido prolificos en normas jurídicas de integración, pero como lo muestra un estudio de un destacado académico argentino, en uno de los esquemas de integración del Mercosur, no más de un 30 por ciento de las normas que se aprobaron dentro de los países forman parte o han formado parte del orden juridico interno de cada país. Esto nos demuestra que tenemos una gran convicción al firmar cosas y después no tan alta al implementarlas.

I a experiencia europea ha sido diferente. Después del término de la Segunda Guerra Mundial, Europa se encontraba devastada por los conflictos bélicos y el 9 de mayo de 19.50) el Ministro de Asuntos Exteriores francés, Robert Schuman, hizo una afirmación muy clara: "Fatropa no se hará de utha lez, se hati gracias a realizaciones concretas, paso a paso". Por eso partieron con la Comunidad del Carbón y del Acero. Yo me vine estos dias al trabajo con algunos colaboradores del Ministerio, Directores muy importantes como Juan Pablo I ira o Carlos Appelgren, y nos dimos el trabajo de mirar las cifras. La verdad es que es muy impresionante lo que ocurrió después de la firma del Tratado del Carbón y del Acero. Fste Tratado significó un aumento impresionante de las fuentes de energía en la producción e inversión en acero para la reconstrucción de la Comunidad Europea, sohre todo, para la integración física.

Si siguiéramos el ejemplo europeo, deberíamos poner urgencia en América Latina a la integración a través del tratamiento común en materia de recursos o necesidades esenciales 
para todos, como son la energía y la infraestructura, pero también convergencia en la equidad social, que es un desafío primordial como expresan Ricardo Ffrench-Davis y Andras Uthoff, en sus respectivos capitulos.

Por ejemplo, si nos ponemos serios en el tema cnergético, tenemos que llegar a algún acuerdo marco, por lo menos, para realizar algo que es obvio y que, sin embargo, no ocurre en la región. Tenemos países excedentarios en recursos energéticos, que tienen verdaderos océanos de gas o de petróleo debajo de la tierra, y tenemos países deficitarios como Brasil, Argentina, Paraguay, Uruguay y Chile, y no nos hemos puesto de acuerdo en un marco que nos permita que los que tienen de sobra le vendan a un precio razonable a los que ticnen un déficit. Para eso, es obvio, las cosas que funcionan y las que no funcionan. Aquí sí, para este tema, necesitamos reglas comunes y un marco jurídico comín, un tratamiento garantizado para la inversión de cualquier país que haga en otro país, una política de precios razonables y compatibles con el escenario internacional y fomentar la asociatividad entre empresas de distintos paises, para lograr así un estricto cumplimiento de los contratos en materia de energía y, ojalá, políticas tributarias armonizadas.

Esto, que parece tan obvio y tan evidente, nosotros ahora en UNASUR lo vamos a tratar como un tema de primera prioridad y vamos a llevar adelante algunas iniciativas con nuestro Ministerio de Energía para, incluso, instalar en Chile un centro de investigación de energías renovables; pero esperamos que nucstras empresas públicas en el campo energético se asocien y trabajen en común, hagan inversiones y avancemos de verdad.

En la integración física, pasa algo parecido. En el año 2000, en Brasilia, los Jefes de Estado se reunieron y lanzaron el Plan IIRSA. Por alguna razón extraña, estaba allá en una reunión anexa, anterior a la reunión de los jefes de Estado y vi la celebración que hubo en Brasilia y en el resto América Latina por cl Plan IIRSA. De nuevo, en un lenguaje más biell académico, me pongo ese sombrero más que el de diplomático. El dia de hoy vengo a ver en qué se ha avanzado, a evaluar los proyectos. ¿Cuántos de los corredores bioceánicos se han terminado? Ninguno. Por eso es que tuvimos la idea y se lo planteamos a lia Presidenta Bachelet y al Presidente Lula cuando vino a Chile 
y les dijimos "tomemos uno de estos corredores biocénicos y' hagámoslon. O sea, concretemos de un lado al otro, y que el Presidente Morales, el Presidente Lula y la Presidenta Bachelet puedan, en un helicóptero o en un vehículo, recorrer desde Santos hasta Iquique o Arica antes del fin de 2010.

Parece simple, pero no ha ocurrido y, en realidad, tenemos que preguntarnos por qué estas cosas no ocurren. Aunque no voy a entrar en eso, porque me voy a alargar mucho.

Respecto del tercer tema: convergencia en política social. Nosotros propusimos en UNASUR, y lo vamos a llevar adelante, como una pequeña muestra de colaboración, establecer un observatorio de políticas sociales y de avances sociales. Así como nuestros economistas aquí y en Wall Street andan pendientes de lo que pasa todos los días con el PIB chileno, con los precios, la inflación, las tasas de interés, sería bastante interesante que las autoridades que hay en los distintos países se vean obligadas a mirar todos los dias las cifras de avances y retrocesos que ocurren en el plano social en otros países y que se sepa, exactamente, cuándo no estamos resolviendo problemas que debiéramos resolver para los sectores más vulnerables, los desempleados crónicos, las Pymes que son, eventualmente, amenazadas por una política cambiaria que no funciona adecuadamente. En fin, un inventario, una fotografía continua, constante, de lo que ocurre en el plano social, porque eso va a obligar a que las instancias superiores de los países tengan que responder continuamente ante la necesidad de resolver las necesidades de nuestra región, como condición para la consolidación de las democracias y como condición para un crecimiento económico sostenido, que vaya más allá de las frustraciones de los ciclos internacionales.

Estas son las tareas concretas que la Preside :ta Bachelet planteó en la reunión fundacional de UNASUR, y que vamos a llevar adelante este año.

Creo que esta postura va en la idea de lo que Schuman recomendaba a los europeos al iniciar su proceso de integración, esto es, tareas concretas, avances medibles, retórica en retirada. Ese es, a nuestro juicio, el camino para recuperar el ticmpo perdido en el proceso de integración regional.

Concuerdo con lo que plantea Juan Gabriel Valdés, en que Chile debe adoptar nuevas estrategias para enfrentar ol 
mevo escenario internacional y terminar, por lo menos en la imagen públicil, con esta especie de disociación que hay en la mente de la gente, de que Chile se volvió un poco loco en hacer Tratados de Libre Comercio y que se olvidó de todo lo demás, y que hay una especic de repechaje para decir «bucno, acordémonos que el desarrollo de un pais y la integración de un pais al mundo es, también, un tema políticon. No hay un factor más determinante e inamovible en la historia de un país que la geografía y, por lo tanto, el tema de la integración regional es un imperativo ineludible que va a tener que tomar en consideración no solo las negociaciones comerciales, si hay o no un acuerdo, si es un TLC o si es un acuerdo base o si es no sé qué, o no sé cuanto. Es un proceso de convergencia política en la que decidimos con otros países compartir el futuro. Compartir el siglo XXI y cerrar la puerta a los viejos antagonismos que nos siguen dividiendo, que crean mal ambiente. Un ambiente de hostilidad, mala onda y que nos impiden mirar hacia el futuro. Ahi, el artículo de Rodríguez Elizondo es muy fundamental en este libro en señalar, precisamente, la complejidad para hacer este giro.

Yo quisiera hacer una breve referencia a que la otra convergencia que tenemos que buscar con nuestros países en la región se refiere al tema de cómo nos hacemos competitivos, cn conjunto con los países de la región, para enfrentar al resto del mundo. La competitividad es un tema que está en discusión todos los días. Recuerdo que un ex Ministro de Singapur decía una vez cómo se han ido acortando los ciclos en que los productores en China son capaces de imitar una innovación que se produce en Singapur. Al principio, el ciclo duraba tres años, después dos años y después un año. Actualmente, a veces, en algunos productos, cuatro meses. Por lo tanto, las personas que están en Singapur en los centros de investigación, los empresarios dicen: «boy, lo que tenemos que hacer es inventar cosas nuevas cada cuatro meses. Poratue lo anterior ya no l'ale, porque China tiene salarios más bajos, porque tiene mucho mayor escala de produccion", y China, a su vez, hizo un trato con Vietnam.

Fntonces, hay que mantenerse a la vanguardia del proceso de innovación y los tiempos se acortan. Por lo tanto, el mensaje central que nosotros queremos transmitir a través de 
UNASUR es lo que la Presidenta dijo el 21 de mayo como un mensaje hacia los chilenos.

Cuando un país -y cste es un punto muy fundamental-dice: "yo tengo un excedente de fondos del cobre y algunos los voy a ahorrar para las pensiones de la gente el día de mañana y otros para tener políticas sociales", significa que ha adoptado una decisión fundamental. El Fondo Bicentenario de Capital Humano es muy relevante. ¿Cuánta plata vamos a poner? Yo fui Ministro de Hacienda y sé cómo uno regatea las platas. Cuando viene un Ministro de Educación a pedir una pequeña ampliación para la educación básica, unas "luquitas" más para la educación secundaria o para la universitaria. Aquí se ha dado un salto gigantesco. Un Fondo de 6 mil millones de dólares para capital humano que, invertido conservadoramente, debiera reunir entre 200 millones de dólares y 240 millones de dólares al año, para gastar exclusivamente en el extranjero en formación de profesionales chilenos al nivel de postgrado, magíster y doctorado; o, al nivel de técnicos de educación técnica profesional, desde las escuelas agrícolas de la SNA, a los institutos profesionales de las regiones, en Inacap, etc. que puedan enviar a hacer prácticas afuera o que puedan completar estudios en temas tan diversos como computación, lechería, metalurgia, diseño, etc.

Realmente, venimos muy estimulados después de estar en Canadá y en California, donde los Jefes de Estado y el Gobernador de California han planteado esta iniciativa, como diciendo: "Esto es lo que tenemos que hacer en conjunto".

Y no los voy a cansar con cifras. Pero, ya tenemos un plan que si los Ministerios respectivos dejan de lado las prácticas un poco burocráticas, debería permitirnos que al año 2012 pudiéramos mandar a 6 mil estudiantes a estudio de postgrado, doctorados y estudios profesionales, y debiéramos estar hiaciendo pasantías, por ejemplo, de profesores a mitad de carrera, en esta universidad o en cualquier otra. Que puedan ir como "fellows" a un centro importante avanzado de investigación.

El concepto que hemos desarrollado en Cancillería es el de "países afines". Nosotros queremos conectar la política exterior con la estrategia de desarrollo, y la estrategia de desarrollo es li competitividad en el proceso de globalización. Y eso es una sola cosa: aprovechar los talentos del país, donde quiera que estén, al 
máximo. Abrir caminos de oportunidades, levantando el techo de nubes para que cada persona desarrolle todo lo que pueda $\mathrm{en}$ términos de creatividad, en función del desarrollo del país.

Entonces, ¿cuáles van a ser los socios? Empezamos con Canadá y California. Estuvimos con Gordon Brown en Inglaterra. Vamos a armar un programa a firmar con Gran Bretaña. Estamos avanzando en algo parecido con Alemania, y me voy a fines de julio a Australia y a Nueva Zelandia, donde vamos a firmar un TLC con Australia -otro más- y, esta vez, queremos armar un consorcio de universidades australianas y ncozelandesas, para entrar en esta misma materia.

Queremos compartir esto cuando estamos hablando de integración, porque queremos que sea algo que nos obligue a los paises de América Latina y de América del Sur, a trabajar en conjunto para levantar el nivel y hacer la interacción entre has universidades de Brasil, de Argentina, de Chile, de Uruguay, donde haya buenos programas de postgrado y poder enviar a nuestros becados y viccversa.

Termino diciendo que el libro subraya otras instancias de integración que hay en la región. La decisión de Chile es participar activamente en todas ellas, como Grupo de Río, Mercosur, Comunidad Andina, UNASUR, etc.

Creo que son interesantes en el libro los aportes de varios artículos de Romeo Pérez, Alfredo Fuentes, Juan Pablo Lira, Félix Peña, sobre algunos de estos esquemas $y$, de nuevo, Rodríguez Elizondo sobre los avances y dificultades en la integración de carácter vecinal.

Está también el trabajo de César Salazar sobre la integración centroamericana. Una subregión con la que tenemos un trabajo importante $y$, en ese sentido, quiero señalar algo que es poco conocido, y es que Chile está haciendo una acción bastante relevante en materia de cooperación horizontal y triangular -en la que inclumos a la Unión Europea, al Gobiemo de Japón, etc.- y tenemos más de 80 proyectos de cooperación técnica de Chile en países de América Latina, un número importante en Centroamérica y otro número importante en los países vecinos.

En las conversaciones con la Unión Europea, planteamos la idea de pasar a una asociación que llamamos "para el desarrollo" y en ella -uno de los puntos centrales- es que quisié- 
ramos trabajar en conjunto con la Unión Furopea proyectos de cooperación én el resto de la región. Ellos tienen bastantes más recursos que nosotros. Nosotros tenemos mucha capacidad técnica y profesional instalada en las universidades $y$ en los centros de investigación, lo que los gringos llaman "practitioners".

La gente que ha estado en los gobiernos este último tiempo riene cosas que aportar y queremos adelantar eso con un aporte de recursos de la Unión Europea.

En todo caso, como dice César Salazar en el libro, las medidas públicas deben tener un claro propósito para mejorar las condiciones de vida de la gente de una manera más equitativa y más justa. Fsto, para nosotros, es una verdadera obsesión y es algo que, además, los Presidentes de la República con los cuales nos ha tocado trabajar desde 1990 para adelante, lo han establecido como la primerísima prioridad -todos ellosen cuanto al objetivo de desarrollo del país.

la idea, como también se dice en un artículo del libro, es construir en cada país el Estado Social y Democrático de Derecho que plantea Pablo Lazo y, ciertamente, caracterizado por dosis fuertes de gobernabilidad, que es esencial para dar adeculada protección a los sectores más débiles, como puntualiza Enrique Correa.

Quiero concluir resaltando el valor de esta iniciativa de la Universidad Miguel de Cervantes que, con el aporte de la Fundación Konrad Adenauer, ha generado el libro «Los Caminos de la Integración de América latina", que contribuye a la reflexión de cómo avanzamos hacia un espacio de solidaridad regional, tal como se expuso en ese ciclo de conferencias.

Para terminar, una reflexión personal: creo que asi como en los cultimos años Anérica Latina y el Caribe han mostrado índices positivos en materia de crecimiento, ahora podemos crear las condiciones para dar un salto más grande en materia de desarrollo. Eso vale para nosotros y para nuestros aliados naturales en la región.

Debemos asumir ahora tareas de liderazgo tanto en UNA. SUR comoen el Grupo de Río. Y' lo haremos con la convicción de que hay que producir acciones concretas para todos los habitantes de cada uno de nuestros países. Menos retórica y más acciones concretas. Trabajar no solo para las próximas 
elecciones, como lo dijo la Presidenta Bachelet en California, sino para las próximas generaciones. Para todos aquellos que, como resalta Héctor Casanueva, muestran una alta valoración del camino conjunto y que anhelan la integración, como son todos nuestros países. Eso lo consideramos una necesidad pragmática y una obligación ética para nuestra generación. 